\title{
THE STANDARD MODEL WITHIN NON-ASSOCIATIVE GEOMETRY
}

\author{
RAIMAR WULKENHAAR
}

\begin{abstract}
Institut Für Theoretische Physik, Universität LeIPZIG Augustusplatz 10/11, D-04109 Leipzig, Germany

E-mail: wulkhaar@tph100.physik.uni-leipzig.de
\end{abstract}

\begin{abstract}
We present the construction of the standard model within the framework of non-associative geometry. For the simplest scalar product we get the tree-level predictions $m_{W}=\frac{1}{2} m_{t}, m_{H}=\frac{3}{2} m_{t}$ and $\sin ^{2} \theta_{W}=\frac{3}{8}$. These relations differ slightly from predictions derived in non-commutative geometry.
\end{abstract}

\section{INTRODUCTION}

One of the most important applications of non-commutative geometry [1] to physics is a unified description of the standard model. The most elegant version rests upon a K-cycle [1, 2] with real structure [3], see [4, 5] for details and [6, 7] for an older version. There also exist numerous other formulations within non-commutative geometry (NCG), see for instance [8, 9]. The author of this paper has proposed in [10] a modification of non-commutative geometry. In that approach one uses unitary Lie algebras instead of unital associative $*$-algebras. Lie algebras are non-associative algebras - this is the motivation for the working title "non-associative geometry". The only realistic physical model that one can construct within the most elegant NCG-prescription is the standard model [11]. The advantage of non-associative geometry is that a larger class of physical models can be constructed from the same amount of structures as in the most elegant NCG-formulation. That class includes the standard model, as we show in this paper.

We give in Section 2 a recipe how to construct classical gauge field theories within non-associative geometry. The arguments why this recipe works can be found in [10]. Section 3 contains the construction of the standard model. We derive the geometric structures and write down the bosonic action for the simplest scalar product. The fermionic action will not be displayed, because it is identical with the classical formulation.

Date: June 17, 2018. 


\section{The Recipe of Non-Associative Geometry}

The basic object in non-associative geometry is an $\mathrm{L}$-cycle $(\mathfrak{g}, h, D, \pi, \Gamma)$, which consists of a $*$-representation $\pi$ of a unitary Lie algebra $\mathfrak{g}$ in bounded operators on a Hilbert space $h$, together with a selfadjoint operator $D$ on $h$ with compact resolvent and a selfadjoint operator $\Gamma$ on $h, \Gamma^{2}=\mathrm{id}_{h}$, which commutes with $\pi(\mathfrak{a})$ and anticommutes with $D$. The operator $D$ may be unbounded, but such that $[D, \pi(\mathfrak{g})]$ is bounded. L-cycles are naturally related to physical models if the following input data are given:

1) The (Lie) group of local gauge transformations $\mathscr{G}$.

2) Chiral fermions $\boldsymbol{\psi}$ transforming under a representation $\tilde{\pi}$ of $\mathscr{G}$.

3) The fermionic mass matrix $\widetilde{\mathcal{M}}$, i.e. fermion masses plus generalized Kobayashi-Maskawa matrices.

4) Possibly the symmetry breaking pattern of $\mathscr{G}$.

Take $\mathfrak{g}=C^{\infty}(X) \otimes \mathfrak{a}$ as the Lie algebra of $\mathscr{G}$, where $\mathfrak{a}$ is a matrix Lie algebra and $C^{\infty}(X)$ the algebra of smooth functions on the (compact Euclidian) spacetime manifold $X$. Take $h=L^{2}(X, S) \otimes \mathbb{C}^{F}$ as the completion of the Euclidian fermions, where $L^{2}(X, S)$ is the Hilbert space of square integrable bispinors. Take $\pi=1 \otimes \hat{\pi}$ as the differential $\tilde{\pi}_{*}$, where $\hat{\pi}$ is a representation of $\mathfrak{a}$ in $\mathrm{M}_{F} \mathbb{C}$. Put $D=\mathrm{D} \otimes \mathbb{1}_{F}+\gamma^{5} \otimes \mathcal{M}$, where $\mathrm{D}$ is the Dirac operator on $X$ and $\mathcal{M} \in \mathrm{M}_{F} \mathbb{C}$ such that $\gamma^{5} \otimes \mathcal{M}$ coincides with $\widetilde{\mathcal{M}}$ on chiral fermions. The chirality properties of the fermions are encoded in $\Gamma=\gamma^{5} \otimes \hat{\Gamma}$.

The recipe towards the (classical) gauge field theory associated to the $\mathrm{L}-$ cycle is the following: Let $\Omega^{1} \mathfrak{a}$ be the space of formal commutators

$$
\omega^{1}=\sum_{\alpha, z \geq 0}\left[a_{\alpha}^{z}, \ldots\left[a_{\alpha}^{1}, d a_{\alpha}^{0}\right] \ldots\right], a_{\alpha}^{i} \in \mathfrak{a}
$$

Apply linear mappings $\hat{\pi}: \Omega^{1} \mathfrak{a} \rightarrow \mathrm{M}_{F} \mathbb{C}$ and $\hat{\sigma}: \Omega^{1} \mathfrak{a} \rightarrow \mathrm{M}_{F} \mathbb{C}$ defined by

$$
\begin{aligned}
\hat{\pi}\left(\omega^{1}\right) & :=\sum_{\alpha, z \geq 0}\left[\hat{\pi}\left(a_{\alpha}^{z}\right), \ldots\left[\hat{\pi}\left(a_{\alpha}^{1}\right),\left[-\mathrm{i} \mathcal{M}, \hat{\pi}\left(a_{\alpha}^{0}\right)\right]\right] \ldots\right] \\
\hat{\sigma}\left(\omega^{1}\right) & :=\sum_{\alpha, z \geq 0}\left[\hat{\pi}\left(a_{\alpha}^{z}\right), \ldots\left[\hat{\pi}\left(a_{\alpha}^{1}\right),\left[\mathcal{M}^{2}, \hat{\pi}\left(a_{\alpha}^{0}\right)\right]\right] \ldots\right]
\end{aligned}
$$

Define $\Omega^{n} \mathfrak{a} \supset \omega^{n}=\sum_{\alpha}\left[\omega_{n, \alpha}^{1},\left[\omega_{n-1, \alpha}^{1}, \ldots\left[\omega_{2, \alpha}^{1}, \omega_{1, \alpha}^{1}\right] \ldots\right]\right]$, where $\omega_{i, \alpha}^{1} \in \Omega^{1} \mathfrak{a}$. Extend $\hat{\pi}$ and $\hat{\sigma}$ recursively to $\Omega^{n} \mathfrak{a}$ by

$$
\begin{aligned}
& \hat{\pi}\left(\left[\omega^{1}, \omega^{k}\right]\right):=\hat{\pi}\left(\omega^{1}\right) \hat{\pi}\left(\omega^{k}\right)-(-1)^{k} \hat{\pi}\left(\omega^{k}\right) \hat{\pi}\left(\omega^{1}\right), \\
& \hat{\sigma}\left(\left[\omega^{1}, \omega^{k}\right]\right):=\hat{\sigma}\left(\omega^{1}\right) \hat{\pi}\left(\omega^{k}\right)-\hat{\pi}\left(\omega^{k}\right) \hat{\sigma}\left(\omega^{1}\right)-\hat{\pi}\left(\omega^{1}\right) \hat{\sigma}\left(\omega^{k}\right)-(-1)^{k} \hat{\sigma}\left(\omega^{k}\right) \hat{\pi}\left(\omega^{1}\right) .
\end{aligned}
$$

Define for $n \geq 2$

$$
\hat{\pi}\left(\mathcal{J}^{n} \mathfrak{a}\right):=\left\{\hat{\sigma}\left(\omega^{n-1}\right), \omega^{n-1} \in \Omega^{n-1} \mathfrak{a} \cap \operatorname{ker} \hat{\pi}\right\}
$$


Define spaces $\mathbb{r}^{0} \mathfrak{a} \subset \mathrm{M}_{F} \mathbb{C}$ and $\mathbb{r}^{1} \mathfrak{a} \subset \mathrm{M}_{F} \mathbb{C}$ elementwise by

$$
\begin{aligned}
\mathbb{r}^{0} \mathfrak{a} & =-\left(\mathbb{r}^{0} \mathfrak{a}\right)^{*}=\hat{\Gamma}\left(\mathbb{r}^{0} \mathfrak{a}\right) \hat{\Gamma}, & \mathbb{r}^{1} \mathfrak{a} & =-\left(\mathbb{r}^{1} \mathfrak{a}\right)^{*}=-\hat{\Gamma}\left(\mathbb{r}^{1} \mathfrak{a}\right) \hat{\Gamma}, \\
{\left[\mathbb{r}^{0} \mathfrak{a}, \hat{\pi}(\mathfrak{a})\right] } & \subset \hat{\pi}(\mathfrak{a}), & & {\left[\mathbb{r}^{0} \mathfrak{a}, \hat{\pi}\left(\Omega^{1} \mathfrak{a}\right)\right] \subset \hat{\pi}\left(\Omega^{1} \mathfrak{a}\right), } \\
\left\{\mathbb{r}^{0} \mathfrak{a}, \hat{\pi}(\mathfrak{a})\right\} & \subset\{\hat{\pi}(\mathfrak{a}), \hat{\pi}(\mathfrak{a})\}+\hat{\pi}\left(\Omega^{2} \mathfrak{a}\right), & & \left\{\mathbb{r}^{0} \mathfrak{a}, \hat{\pi}\left(\Omega^{1} \mathfrak{a}\right)\right\} \subset\left\{\hat{\pi}(\mathfrak{a}), \hat{\pi}\left(\Omega^{1} \mathfrak{a}\right)\right\}+\hat{\pi}\left(\Omega^{3} \mathfrak{a}\right), \\
{\left[\mathbb{r}^{1} \mathfrak{a}, \hat{\pi}(\mathfrak{a})\right] } & \subset \hat{\pi}\left(\Omega^{1} \mathfrak{a}\right), & & \left\{\mathbb{r}^{1} \mathfrak{a}, \hat{\pi}\left(\Omega^{1} \mathfrak{a}\right)\right\} \subset \hat{\pi}\left(\Omega^{2} \mathfrak{a}\right)+\{\hat{\pi}(\mathfrak{a}), \hat{\pi}(\mathfrak{a})\} .
\end{aligned}
$$

Define spaces $j^{0} \mathfrak{a}, \dot{j}^{1} \mathfrak{a}, \dot{j}^{2} \mathfrak{a} \subset \mathrm{M}_{F} \mathbb{C}$ elementwise by

$$
\begin{aligned}
& \dot{j}^{0} \mathfrak{a}=-\left(\dot{j}^{0} \mathfrak{a}\right)^{*}=\hat{\Gamma}\left(\dot{j}^{0} \mathfrak{a}\right) \hat{\Gamma}, \quad \dot{j}^{1} \mathfrak{a}=-\left(\dot{j}^{0} \mathfrak{a}\right)^{*}=-\hat{\Gamma}\left(\dot{j}^{0} \mathfrak{a}\right) \hat{\Gamma}, \quad \dot{j}^{2} \mathfrak{a}=\left(\dot{j}^{0} \mathfrak{a}\right)^{*}=\hat{\Gamma}\left(\dot{j}^{0} \mathfrak{a}\right) \hat{\Gamma} \\
& {\left[\dot{j}^{0} \mathfrak{a}, \hat{\pi}(\mathfrak{a})\right]=0, \quad\left\{\dot{j}^{0} \mathfrak{a}, \hat{\pi}(\mathfrak{a})\right\} \subset \hat{\pi}\left(\mathcal{J}^{2} \mathfrak{a}\right)+\{\hat{\pi}(\mathfrak{a}), \hat{\pi}(\mathfrak{a})\}} \\
& {\left[\dot{j}^{0} \mathfrak{a}, \hat{\pi}\left(\Omega^{1} \mathfrak{a}\right)\right]=0, \quad\left\{\dot{j}^{0} \mathfrak{a}, \hat{\pi}\left(\Omega^{1} \mathfrak{a}\right)\right\} \subset \hat{\pi}\left(\mathcal{J}^{3} \mathfrak{a}\right)+\left\{\hat{\pi}\left(\Omega^{1} \mathfrak{a}\right), \hat{\pi}(\mathfrak{a})\right\},} \\
& {\left[\dot{j}^{1} \mathfrak{a}, \hat{\pi}(\mathfrak{a})\right]=0, \quad\left\{\dot{j}^{1} \mathfrak{a}, \hat{\pi}\left(\Omega^{1} \mathfrak{a}\right)\right\} \subset \hat{\pi}\left(\mathcal{J}^{2} \mathfrak{a}\right)+\{\hat{\pi}(\mathfrak{a}), \hat{\pi}(\mathfrak{a})\},} \\
& \left\{\dot{j}^{1} \mathfrak{a}, \hat{\pi}(\mathfrak{a})\right\} \subset \hat{\pi}\left(\mathcal{J}^{3} \mathfrak{a}\right)+\left\{\hat{\pi}\left(\Omega^{1} \mathfrak{a}\right), \hat{\pi}(\mathfrak{a})\right\} \\
& {\left[\dot{j}^{1} \mathfrak{a}, \hat{\pi}\left(\Omega^{1} \mathfrak{a}\right)\right] \subset \hat{\pi}\left(\mathcal{J}^{4} \mathfrak{a}\right)+\left\{\hat{\pi}\left(\Omega^{2} \mathfrak{a}\right)+\{\hat{\pi}(\mathfrak{a}), \hat{\pi}(\mathfrak{a})\}, \hat{\pi}(\mathfrak{a})\right\}+} \\
& +\left[\hat{\pi}\left(\Omega^{1} \mathfrak{a}\right),\{\hat{\pi}(\mathfrak{a}), \hat{\pi}(\mathfrak{a})\}\right]+\hat{\sigma}\left(\hat{\pi}^{-1}\left(\left\{\hat{\pi}(\mathfrak{a}), \hat{\pi}\left(\Omega^{1} \mathfrak{a}\right)\right\} \cap \hat{\pi}\left(\Omega^{3} \mathfrak{a}\right)\right)\right), \\
& {\left[\dot{j}^{2} \mathfrak{a}, \hat{\pi}(\mathfrak{a})\right] \subset \hat{\pi}\left(\mathcal{J}^{2} \mathfrak{a}\right)+\{\hat{\pi}(\mathfrak{a}), \hat{\pi}(\mathfrak{a})\}, \quad\left[\dot{j}^{2} \mathfrak{a}, \hat{\pi}\left(\Omega^{1} \mathfrak{a}\right)\right] \subset \hat{\pi}\left(\mathcal{J}^{3} \mathfrak{a}\right)+\left\{\hat{\pi}\left(\Omega^{1} \mathfrak{a}\right), \hat{\pi}(\mathfrak{a})\right\}}
\end{aligned}
$$

The connection form $\rho$ has the structure

$$
\rho=\sum_{\alpha}\left(c_{\alpha}^{1} \otimes m_{\alpha}^{0}+c_{\alpha}^{0} \gamma^{5} \otimes m_{\alpha}^{1}\right), c_{\alpha}^{1} \in \Lambda^{1}, c_{\alpha}^{0} \in \Lambda^{0}, m_{\alpha}^{0} \in \mathbb{r}^{0} \mathfrak{a}, m_{\alpha}^{1} \in \mathbb{r}^{1} \mathfrak{a}
$$

where $\Lambda^{k}$ is the space of differential $k$-forms represented by gamma matrices. The curvature $\theta$ is computed from the connection form $\rho$ by

$$
\begin{aligned}
\theta & =\mathbf{d} \rho+\rho^{2}-\mathrm{i}\left\{\gamma^{5} \otimes \mathcal{M}, \rho\right\}+\hat{\sigma}(\rho) \gamma^{5}+\mathbb{J}^{2} \mathfrak{g}, \\
\mathbb{J}^{2} \mathfrak{g} & =\left(\Lambda^{2} \otimes \mathfrak{j}^{0} \mathfrak{a}\right) \oplus\left(\Lambda^{1} \gamma^{5} \otimes \dot{j}^{1} \mathfrak{a}\right) \oplus\left(\Lambda^{0} \otimes \mathfrak{j}^{2} \mathfrak{a}\right),
\end{aligned}
$$

where $\mathbf{d}$ is the exterior differential and $\hat{\sigma}$ the extension to elements of the form (6). Select the representative $\mathfrak{e}(\theta)$ orthogonal to $\mathbb{J}^{2} \mathfrak{g}$, i.e. find $\mathrm{j} \in \mathbb{J}^{2} \mathfrak{g}$ such that

$$
\mathfrak{e}(\theta)=\mathbf{d} \rho+\rho^{2}-\mathrm{i}\left\{\gamma^{5} \otimes \mathcal{M}, \rho\right\}+\hat{\sigma}(\rho) \gamma^{5}+\mathrm{j}, \quad \int_{X} d x \operatorname{tr}\left(\mathfrak{e}(\theta) j^{2}\right)=0 \forall j^{2} \in \mathbb{J}^{2} \mathfrak{g} .
$$

The trace includes the trace in $\mathrm{M}_{F} \mathbb{C}$ and over gamma matrices. Compute the bosonic and fermionic actions

$$
S_{B}=\int_{X} d x \frac{1}{g_{0}^{2} F} \operatorname{tr}\left(\mathfrak{e}(\theta)^{2}\right), \quad S_{F}=\int_{X} d x \psi^{*}(D+\mathrm{i} \rho) \boldsymbol{\psi}
$$

where $g_{0}$ is a coupling constant and $\boldsymbol{\psi} \in h$. Finally, perform a Wick rotation to Minkowski space. 


\section{The Construction}

Our constructions requires that the mass matrices of all fermions of the same type, including the neutrinos, are different from zero and non-degenerated. In particular, the Kobayashi-Maskawa matrix in both the quark and the lepton sector must be non-trivial. This is necessary to avoid certain degeneracy effects. The matrix Lie algebra of the standard model is

$$
\mathfrak{a}=\operatorname{su}(3) \oplus \operatorname{su}(2) \oplus \mathrm{u}(1) .
$$

The Hilbert space is $\mathbb{C}^{48}$, because we need right neutrinos. We label elements of $\mathbb{C}^{48}$ in a suggestive way by the fermions of the first generation:

$$
\left(\boldsymbol{u}_{L}, \boldsymbol{d}_{L}, \boldsymbol{u}_{R}, \boldsymbol{d}_{R}, \nu_{L}, e_{L}, \nu_{R}, e_{R}\right)^{T} \in \mathbb{C}^{48},
$$

where $\boldsymbol{u}_{L}, \boldsymbol{d}_{L}, \boldsymbol{u}_{R}, \boldsymbol{d}_{R} \in \mathbb{C}^{3} \otimes \mathbb{C}^{3}$ and $\nu_{L}, e_{L}, \nu_{R}, e_{R} \in \mathbb{C}^{3}$. The representation $\hat{\pi}$ of $\mathfrak{a}$ on $\mathbb{C}^{48}$ is

$$
\begin{aligned}
& \hat{\pi}\left(\left(a_{1}, a_{2}, a_{3}\right)\right)= \\
& \text { if } f_{0} \operatorname{diag}\left(\frac{1}{3} \mathbb{1}_{3} \otimes \mathbb{1}_{3}, \frac{1}{3} \mathbb{1}_{3} \otimes \mathbb{1}_{3}, \frac{4}{3} \mathbb{1}_{3} \otimes \mathbb{1}_{3},-\frac{2}{3} \mathbb{1}_{3} \otimes \mathbb{1}_{3},-\mathbb{1}_{3},-\mathbb{1}_{3}, 0_{3},-2 \mathbb{1}_{3}\right)+
\end{aligned}
$$

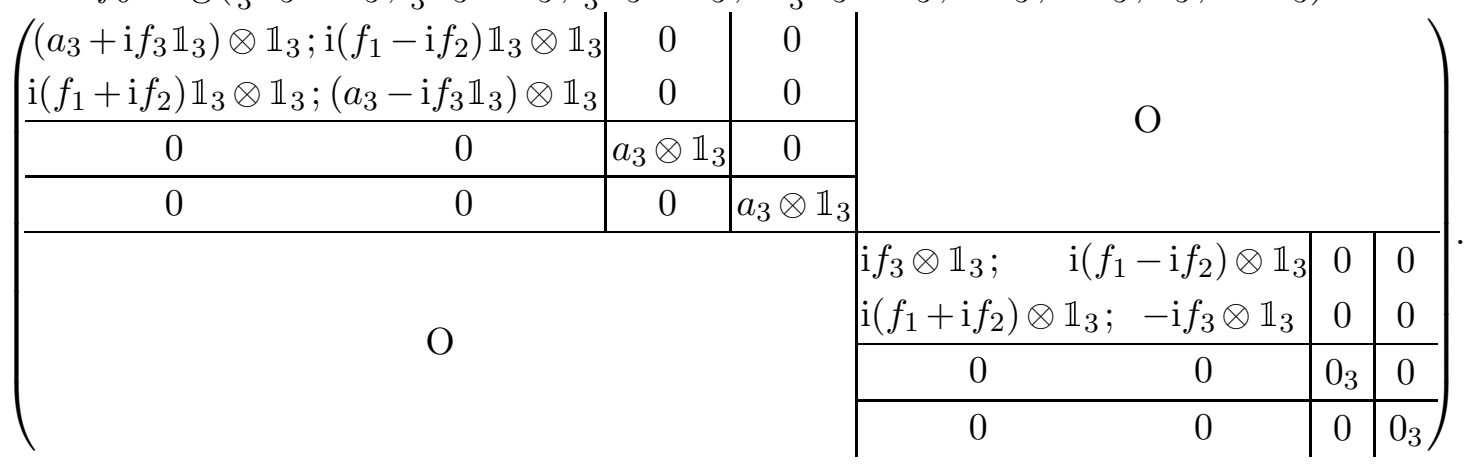

Here, the matrix $a_{3} \in \mathrm{su}(3) \subset \mathrm{M}_{3} \mathbb{C}$ is written down in the standard matrix representation, $a_{2}=\left(\begin{array}{cr}\mathrm{i} f_{3} ; & \mathrm{i}\left(f_{1}-\mathrm{i} f_{2}\right) \\ \mathrm{i}\left(f_{1}+\mathrm{i} f_{2}\right) ; & -\mathrm{i} f_{3}\end{array}\right) \in \mathrm{su}(2)$, for $f_{1}, f_{2}, f_{3} \in \mathbb{R}$, and $a_{1}=\mathrm{i} f_{0} \in$ $\mathrm{u}(1) \equiv \mathrm{i} \mathbb{R}$. The generalized Dirac operator is

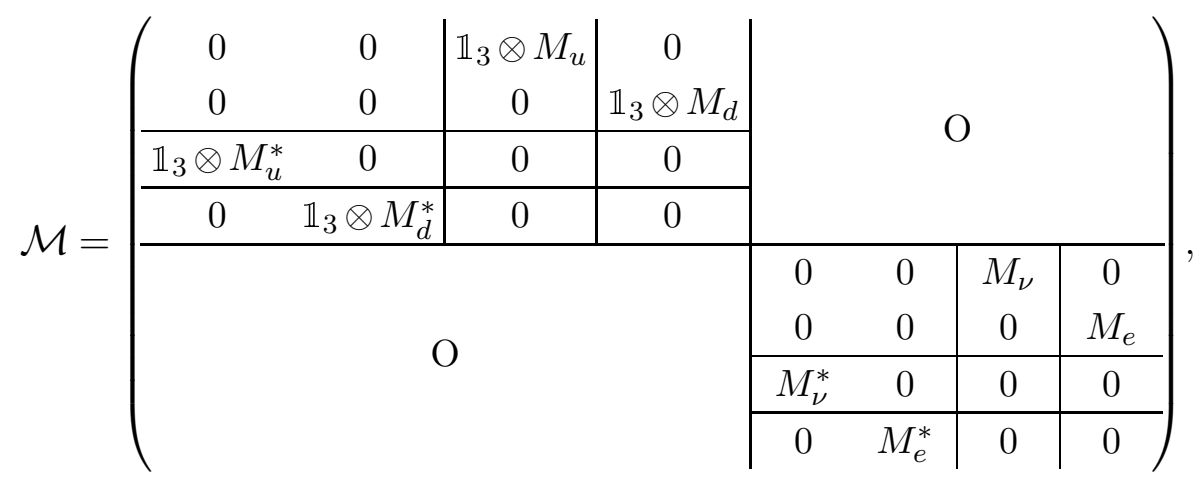


where $M_{u}, M_{d}, M_{\nu}, M_{e} \in \mathrm{M}_{3} \mathbb{C}$ are the mass matrices of the fermions. It is easy to see that for $a_{\alpha}^{i}=\left(a_{3, \alpha}^{i}, a_{2, \alpha}^{i}, a_{3, \alpha}^{i}\right) \in \mathfrak{a}$ one has

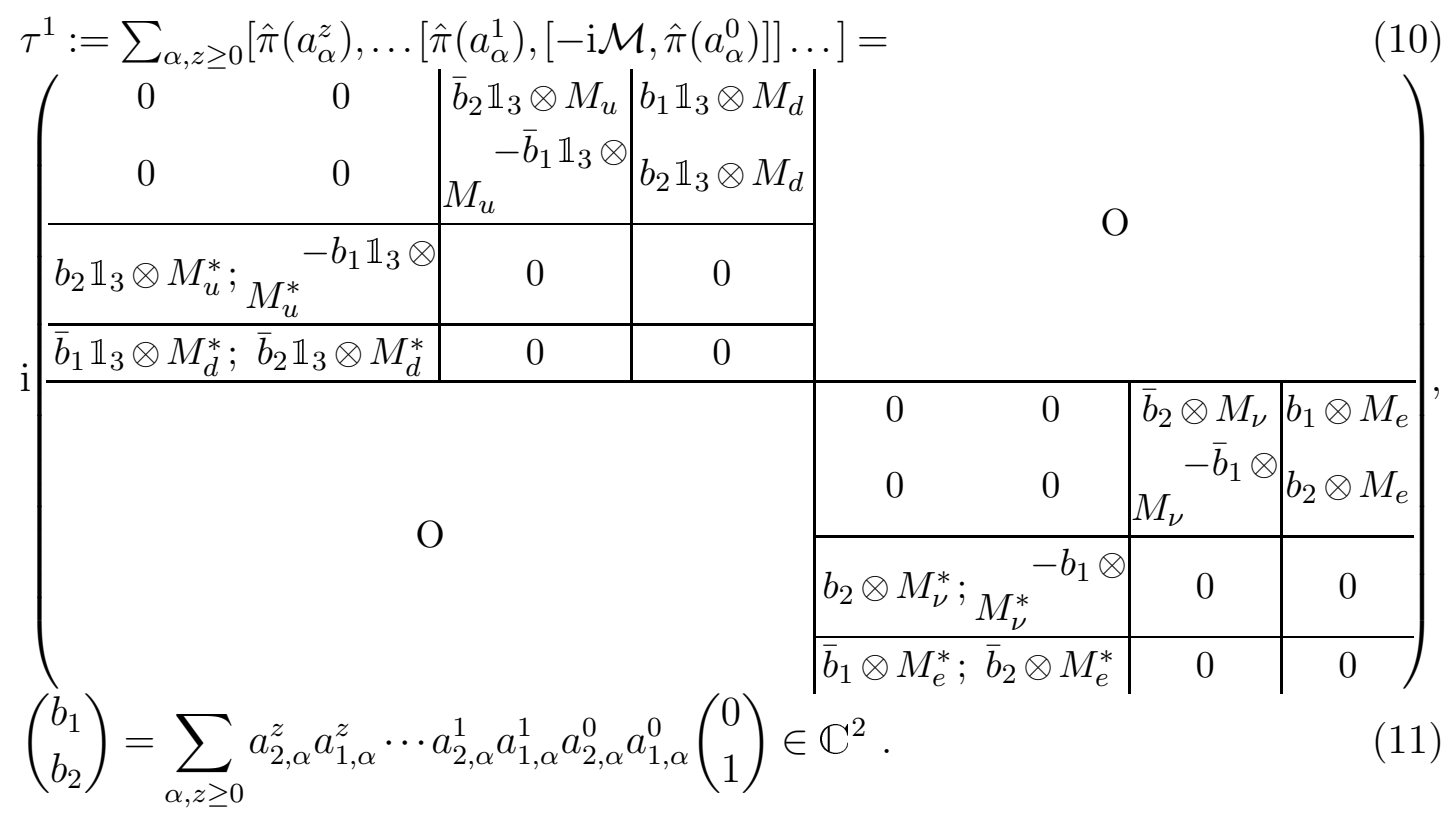

The matrix (10) is the general form of an element of $\hat{\pi}\left(\Omega^{1} \mathfrak{a}\right)$. The grading operator is

$$
\hat{\Gamma}=\operatorname{diag}\left(-\mathbb{1}_{3} \otimes \mathbb{1}_{3},-\mathbb{1}_{3} \otimes \mathbb{1}_{3}, \mathbb{1}_{3} \otimes \mathbb{1}_{3}, \mathbb{1}_{3} \otimes \mathbb{1}_{3},-\mathbb{1}_{3},-\mathbb{1}_{3}, \mathbb{1}_{3}, \mathbb{1}_{3}\right) .
$$

One has $\hat{\Gamma}^{2}=\mathbb{1}_{48},[\hat{\Gamma}, \hat{\pi}(\mathfrak{a})]=0,\{\hat{\Gamma}, \mathcal{M}\}=0$ and $\left\{\hat{\Gamma}, \hat{\pi}\left(\Omega^{1} \mathfrak{a}\right)\right\}=0$. Let

$$
\begin{aligned}
& \left(\begin{array}{lr}
\mathrm{i} f_{3} ; & \mathrm{i}\left(f_{1}-\mathrm{i} f_{2}\right) \\
\mathrm{i}\left(f_{1}+\mathrm{i} f_{2}\right) ; & -\mathrm{i} f_{3}
\end{array}\right):=\left(\begin{array}{cr}
\mathrm{i}\left(\left|b_{2}\right|^{2}-\left|b_{1}\right|^{2}\right) ; & -2 \mathrm{i} b_{1} \bar{b}_{2} \\
-2 \mathrm{i} \bar{b}_{1} b_{2} ; & -\mathrm{i}\left(\left|b_{2}\right|^{2}-\left|b_{1}\right|^{2}\right)
\end{array}\right) \in \mathrm{su}(2), \\
& M_{u d}=M_{u} M_{u}^{*}-M_{d} M_{d}^{*}, M_{\nu e}=M_{\nu} M_{\nu}^{*}-M_{e} M_{e}^{*}, \\
& M_{\{u d\}}=M_{u} M_{u}^{*}+M_{d} M_{d}^{*}, M_{\{\nu e\}}=M_{\nu} M_{\nu}^{*}+M_{e} M_{e}^{*} .
\end{aligned}
$$

Then we have

$\left\{\tau^{1}, \tau^{1}\right\}=$

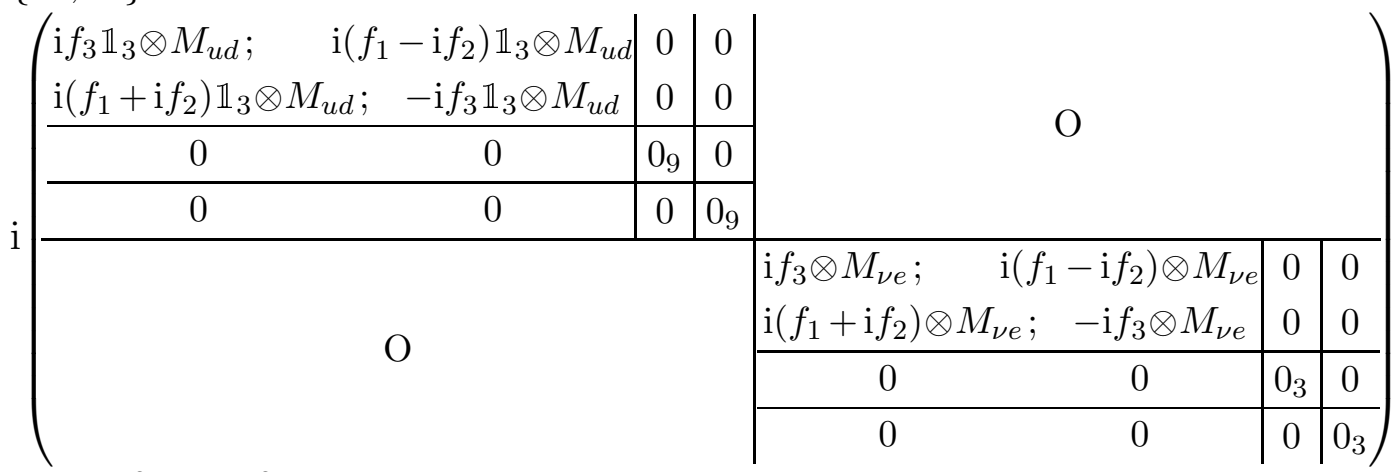

$$
\begin{aligned}
& -\left(\left\|b_{1}\right\|^{2}+\left\|b_{2}\right\|^{2}\right) \operatorname{diag}\left(\mathbb{1}_{3} \otimes M_{\{u d\}}, \mathbb{1}_{3} \otimes M_{\{u d\}}, \mathbb{1}_{3} \otimes 2 M_{u}^{*} M_{u}, \mathbb{1}_{3} \otimes 2 M_{d}^{*} M_{d},\right. \\
& \left.M_{\{\nu e\}}, M_{\{\nu e\}}, 2 M_{\nu}^{*} M_{\nu}, 2 M_{e}^{*} M_{e}\right) .
\end{aligned}
$$


Next, for $\tau^{1}=\hat{\pi}\left(\omega^{1}\right)$ given by (10) we obtain with (21)

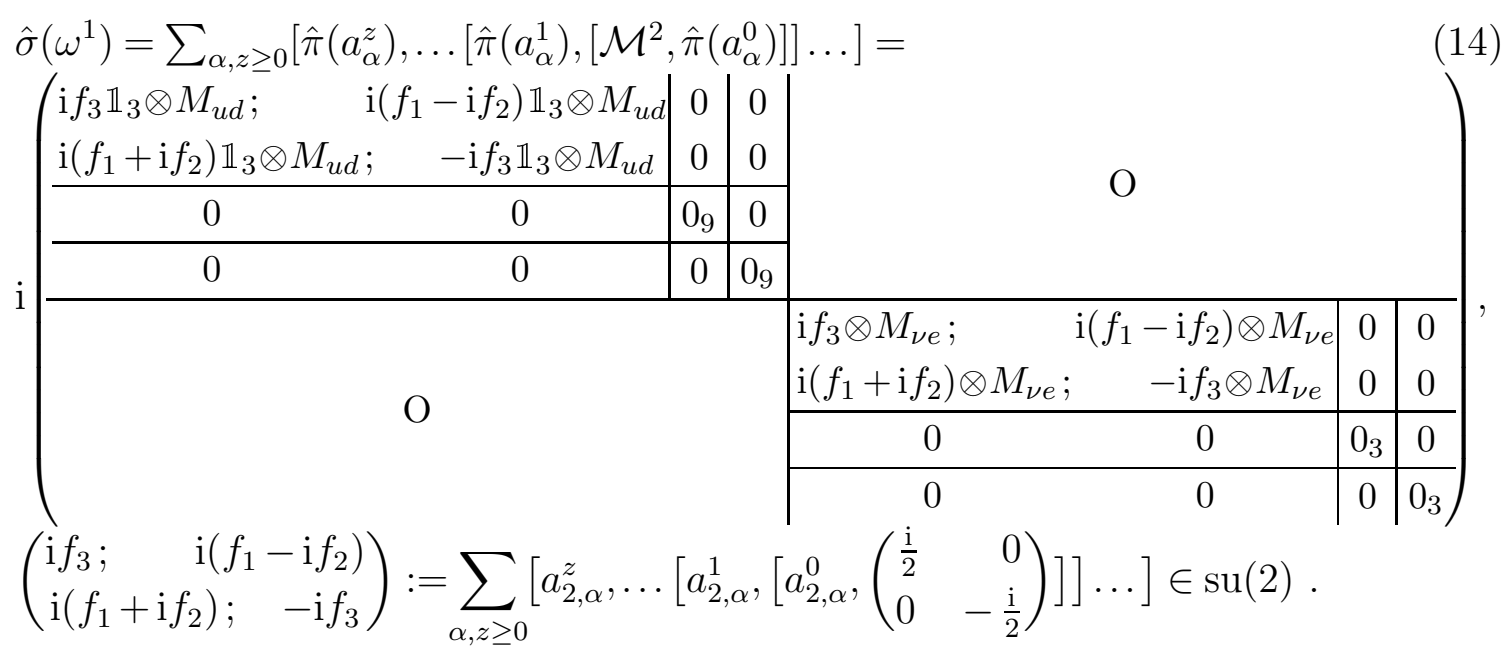

Choosing

$$
\omega_{0}^{1}=d a_{2}^{0}+\left[a_{2}^{1},\left[a_{2}^{1}, d a_{2}^{0}\right], a_{2}^{0}=\left(\begin{array}{cc}
0 & \mathrm{i} \\
\mathrm{i} & 0
\end{array}\right) \in \mathrm{su}(2), a_{2}^{1}=\left(\begin{array}{rr}
\mathrm{i} & 0 \\
0 & -\mathrm{i}
\end{array}\right) \in \mathrm{su}(2),\right.
$$

we have $\omega_{0}^{1} \in \operatorname{ker} \hat{\pi}$ due to $\left(a_{2}^{0}+a_{2}^{1} a_{2}^{1} a_{2}^{0}\right)\left(\begin{array}{l}0 \\ 1\end{array}\right)=\left(\begin{array}{l}0 \\ 0\end{array}\right)$, see (11). On the other hand, $\hat{\sigma}\left(\omega^{1}\right) \neq 0$ is the matrix (14), with $f_{1}=0, f_{2}=-3, f_{3}=0$. Obviously, each matrix of the form (14) can be represented as $\hat{\sigma}\left(\omega^{1}\right)$, for $\omega^{1}=\sum_{\alpha, z \geq 0}\left[a_{\alpha}^{z}, \ldots\left[a_{\alpha}^{0}, \omega_{0}^{1}\right] \ldots\right] \in$ ker $\hat{\pi}$. Therefore, each element of $\hat{\pi}\left(\mathcal{J}^{2} \mathfrak{a}\right)$ is precisely of the form (14), see (3):

$$
\hat{\sigma}\left(\Omega^{1} \mathfrak{a}\right) \equiv \hat{\pi}\left(\mathcal{J}^{2} \mathfrak{a}\right)
$$

Comparing the results (15) and (14) with (12) and (13) we get

$$
\begin{array}{r}
\left\{\tau^{1}, \tau^{1}\right\}=-\left(\left\|b_{1}\right\|^{2}+\left\|b_{2}\right\|^{2}\right) \operatorname{diag}\left(\mathbb{1}_{3} \otimes M_{\{u d\}}, \mathbb{1}_{3} \otimes M_{\{u d\}}, \mathbb{1}_{3} \otimes 2 M_{u}^{*} M_{u}, \mathbb{1}_{3} \otimes 2 M_{d}^{*} M_{d},\right. \\
\left.M_{\{\nu e\}}, M_{\{\nu e\}}, 2 M_{\nu}^{*} M_{\nu}, 2 M_{e}^{*} M_{e}\right) \bmod \hat{\pi}\left(\mathcal{J}^{2} \mathfrak{a}\right) .(16)
\end{array}
$$

It is clear that (16) is orthogonal to $\hat{\pi}\left(\mathcal{J}^{2} \mathfrak{a}\right)$.

Next, we need the structure of the space $\{\hat{\pi}(\mathfrak{a}), \hat{\pi}(\mathfrak{a})\}$. A simple calculation yields for elements of $\{\hat{\pi}(\mathfrak{a}), \hat{\pi}(\mathfrak{a})\}$ the form

$$
\begin{aligned}
& \{\hat{\pi}(\mathfrak{a}), \hat{\pi}(\mathfrak{a})\} \ni \operatorname{diag}\left(A_{q}+\Delta_{q}, A_{\ell}+\Delta_{\ell}\right),
\end{aligned}
$$

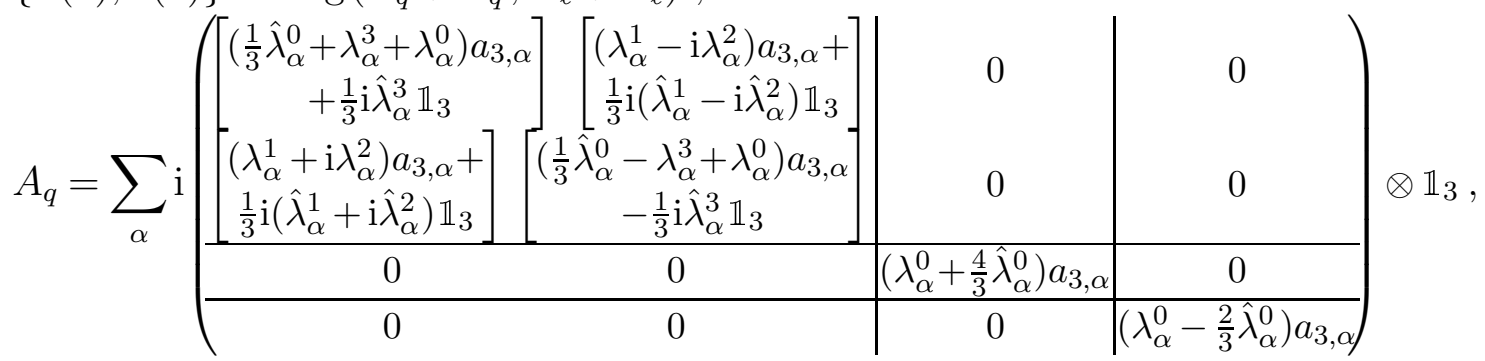




$$
\begin{aligned}
A_{\ell} & =\sum_{\alpha} \mathrm{i}\left(\begin{array}{cc|c|c}
-\mathrm{i} \hat{\lambda}_{\alpha}^{3} \mathbb{1}_{3} ; & -\mathrm{i}\left(\hat{\lambda}_{\alpha}^{1}-\mathrm{i} \hat{\lambda}_{\alpha}^{2}\right) \mathbb{1}_{3} & 0 & 0 \\
-\mathrm{i}\left(\hat{\lambda}_{\alpha}^{1}+\mathrm{i} \hat{\lambda}_{\alpha}^{2}\right) \mathbb{1}_{3} & \mathrm{i} \hat{\lambda}_{\alpha}^{3} \mathbb{1}_{3} & 0 & 0 \\
\hline 0 & 0 & 0_{3} & 0 \\
\hline 0 & 0 & 0_{3}
\end{array}\right), \\
\Delta_{q} & =\operatorname{diag}\left(\left(\lambda+\tilde{\lambda}+\frac{1}{9} \hat{\lambda}\right) \mathbb{1}_{3},\left(\lambda+\tilde{\lambda}+\frac{1}{9} \hat{\lambda}\right) \mathbb{1}_{3},\left(\lambda+\frac{16}{9} \hat{\lambda}\right) \mathbb{1}_{3},\left(\lambda+\frac{4}{9} \hat{\lambda}\right) \mathbb{1}_{3}\right) \otimes \mathbb{1}_{3}, \\
\Delta_{\ell} & =\operatorname{diag}\left((\tilde{\lambda}+\hat{\lambda}) \mathbb{1}_{3},(\tilde{\lambda}+\hat{\lambda}) \mathbb{1}_{3}, 0_{3}, 4 \hat{\lambda} \mathbb{1}_{3}\right),
\end{aligned}
$$

where $a_{3, \alpha} \in \operatorname{su}(3)$ and $\lambda_{\alpha}^{0}, \lambda_{\alpha}^{1}, \lambda_{\alpha}^{2}, \lambda_{\alpha}^{3}, \hat{\lambda}_{\alpha}^{0}, \hat{\lambda}_{\alpha}^{1}, \hat{\lambda}_{\alpha}^{2}, \hat{\lambda}_{\alpha}^{3}, \lambda, \tilde{\lambda}, \hat{\lambda} \in \mathbb{R}$.

In order to write down the structure of the connection form we must find the spaces $\mathbb{r}^{0} \mathfrak{a}$ and $\mathbb{r}^{1} \mathfrak{a}$, see (6). The evaluation of (田) yields in the case of generic mass matrices $M_{u}, M_{d}, M_{\nu}, M_{e}$ the simple result

$$
\mathbb{r}^{0} \mathfrak{a}=\hat{\pi}(\mathfrak{a}), \quad \quad \mathbb{r}^{1} \mathfrak{a}=\hat{\pi}\left(\Omega^{1} \mathfrak{a}\right) .
$$

For generic mass matrices, equations (5) have the solution $\dot{\mathfrak{j}}^{0} \mathfrak{a}=0, \dot{\mathfrak{j}}^{1} \mathfrak{a}=0$ and

$$
\begin{aligned}
\dot{\mathfrak{j}}^{2} \mathfrak{a} & =\hat{\pi}\left(\mathcal{J}^{2} \mathfrak{a}\right) \oplus\{\hat{\pi}(\mathfrak{a}), \hat{\pi}(\mathfrak{a})\} \oplus \operatorname{diag}\left(\mathbb{R}_{18}, \mathbb{R}_{18}, \mathbb{R}_{6}, \mathbb{R}_{1}\right) \\
& \subset J_{2} \oplus \operatorname{diag}\left(A_{q}, A_{\ell}\right) \oplus \operatorname{diag}\left(J_{q}, J_{\ell}\right), \\
J_{q} & =\operatorname{diag}\left(\left(\lambda_{1}+\frac{1}{9} \lambda_{0}\right) \mathbb{1}_{3},\left(\lambda_{1}+\frac{1}{9} \lambda_{0}\right) \mathbb{1}_{3},\left(\lambda_{2}+\frac{16}{9} \lambda_{0}\right) \mathbb{1}_{3},\left(\lambda_{2}+\frac{4}{9} \lambda_{0}\right) \mathbb{1}_{3}\right) \otimes \mathbb{1}_{3}, \\
J_{\ell} & =\operatorname{diag}\left(\left(\lambda_{3}+\lambda_{0}\right) \mathbb{1}_{3},\left(\lambda_{3}+\lambda_{0}\right) \mathbb{1}_{3}, \lambda_{4} \mathbb{1}_{3},\left(\lambda_{4}+4 \lambda_{0}\right) \mathbb{1}_{3}\right),
\end{aligned}
$$

for $J_{2} \in \hat{\pi}\left(\mathcal{J}^{2} \mathfrak{a}\right)$ and $\lambda_{1}, \lambda_{2}, \lambda_{3}, \lambda_{4} \in \mathbb{R}$.

In order to write down the bosonic action it is necessary to select the representative $\mathfrak{e}\left(\left\{\tau^{1}, \tau^{1}\right\}\right)$ of $\left\{\tau^{1}, \tau^{1}\right\}+\dot{\mathfrak{j}}^{2} \mathfrak{a}$ orthogonal to $\dot{j}^{2} \mathfrak{a}$. This problem is easy to solve. Let

$$
\begin{aligned}
\tilde{M}_{\{u d\}} & :=M_{u} M_{u}^{*}+M_{d} M_{d}^{*}-\frac{1}{3} \operatorname{tr}\left(M_{u} M_{u}^{*}+M_{d} M_{d}^{*}\right) \mathbb{1}_{3}, \\
\tilde{M}_{\{\nu e\}} & :=M_{\nu} M_{\nu}^{*}+M_{e} M_{e}^{*}-\frac{1}{3} \operatorname{tr}\left(M_{\nu} M_{\nu}^{*}+M_{e} M_{e}^{*}\right) \mathbb{1}_{3}, \\
\tilde{M}_{u u} & :=M_{u}^{*} M_{u}-\frac{1}{24} \operatorname{tr}\left(5 M_{u} M_{u}^{*}+3 M_{d} M_{d}^{*}-M_{\nu} M_{\nu}^{*}+M_{e} M_{e}^{*}\right) \mathbb{1}_{3}, \\
\tilde{M}_{d d} & :=M_{d}^{*} M_{d}-\frac{1}{24} \operatorname{tr}\left(3 M_{u} M_{u}^{*}+5 M_{d} M_{d}^{*}+M_{\nu} M_{\nu}^{*}-M_{e} M_{e}^{*}\right) \mathbb{1}_{3}, \\
\tilde{M}_{\nu \nu} & :=M_{\nu}^{*} M_{\nu}-\frac{1}{24} \operatorname{tr}\left(-3 M_{u} M_{u}^{*}+3 M_{d} M_{d}^{*}+7 M_{\nu} M_{\nu}^{*}+M_{e} M_{e}^{*}\right) \mathbb{1}_{3}, \\
\tilde{M}_{e e} & :=M_{e}^{*} M_{e}-\frac{1}{24} \operatorname{tr}\left(3 M_{u} M_{u}^{*}-3 M_{d} M_{d}^{*}+M_{\nu} M_{\nu}^{*}+7 M_{e} M_{e}^{*}\right) \mathbb{1}_{3} .
\end{aligned}
$$

Then, the canonical embedding $\mathfrak{e}\left(\left\{\tau^{1}, \tau^{1}\right\}\right)$ of $\left\{\tau^{1}, \tau^{1}\right\}$ into $\mathrm{M}_{48} \mathbb{C}$ is given by $\mathfrak{e}\left(\left\{\tau^{1}, \tau^{1}\right\}\right)=-\left(\left\|b_{1}\right\|^{2}+\left\|b_{2}\right\|^{2}\right) \operatorname{diag}\left(\mathbb{1}_{3} \otimes \tilde{M}_{\{u d\}}, \mathbb{1}_{3} \otimes \tilde{M}_{\{u d\}}, \mathbb{1}_{3} \otimes 2 \tilde{M}_{u u}, \mathbb{1}_{3} \otimes 2 \tilde{M}_{d d}\right.$,

$$
\left.\tilde{M}_{\{\nu e\}}, \tilde{M}_{\{\nu e\}}, 2 \tilde{M}_{\nu \nu}, 2 \tilde{M}_{e e}\right) \text {. }
$$

Now we include the four dimensional Riemannian spin manifold $X$ and choose a selfadjoint local basis $\left\{\gamma^{\mu}\right\}_{\mu=1,2,3,4}$ of $\Lambda^{1}$. The connection form $\rho$ has due to (6), (9) and (10) the structure

$$
\rho=\left(\begin{array}{cc}
\rho_{q} & 0 \\
0 & \rho_{\ell}
\end{array}\right),
$$




$$
\begin{aligned}
\rho_{q} & =\left(\begin{array}{cc|c|c}
\left(\mathbf{A}+\mathrm{i}\left(\frac{1}{3} A^{0}+A^{3}\right) \mathbb{1}_{3}\right) \otimes \mathbb{1}_{3} ; & \mathrm{i}\left(A^{1}-\mathrm{i} A^{2}\right) \mathbb{1}_{3} \otimes \mathbb{1}_{3} & -\mathrm{i} \gamma^{5} \bar{\Phi}_{2} \mathbb{1}_{3} \otimes M_{u} & -\mathrm{i} \gamma^{5} \Phi_{1} \mathbb{1}_{3} \otimes M_{d} \\
\mathrm{i}\left(A^{1}+\mathrm{i} A^{2}\right) \mathbb{1}_{3} \otimes \mathbb{1}_{3} ; & \left(\mathbf{A}+\mathrm{i}\left(\frac{1}{3} A^{0}-A^{3}\right) \mathbb{1}_{3}\right) \otimes \mathbb{1}_{3} & \mathrm{i} \gamma^{5} \bar{\Phi}_{1} \mathbb{1}_{3} \otimes M_{u} & -\mathrm{i} \gamma^{5} \Phi_{2} \mathbb{1}_{3} \otimes M_{d} \\
\hline-\mathrm{i} \gamma^{5} \Phi_{2} \mathbb{1}_{3} \otimes M_{u}^{*} & \mathrm{i} \gamma^{5} \Phi_{1} \mathbb{1}_{3} \otimes M_{u}^{*} & \left(\mathbf{A}+\frac{4}{3} \mathrm{i} A^{0} \mathbb{1}_{3}\right) \otimes \mathbb{1}_{3} & 0 \\
\hline-\mathrm{i} \gamma^{5} \bar{\Phi}_{1} \mathbb{1}_{3} \otimes M_{d}^{*} & -\mathrm{i} \gamma^{5} \bar{\Phi}_{2} \mathbb{1}_{3} \otimes M_{d}^{*} & 0 & \left(\mathbf{A}-\frac{2}{3} \mathrm{i} A^{0} \mathbb{1}_{3}\right) \otimes \mathbb{1}_{3}
\end{array}\right), \\
\rho_{\ell} & =\left(\begin{array}{cc|c|c}
\mathrm{i}\left(-A^{0}+A^{3}\right) \otimes \mathbb{1}_{3} & \mathrm{i}\left(A^{1}-\mathrm{i} A^{2}\right) \otimes \mathbb{1}_{3} & -\mathrm{i} \gamma^{5} \bar{\Phi}_{2} \otimes M_{\nu} & -\mathrm{i} \gamma^{5} \Phi_{1} \otimes M_{e} \\
\mathrm{i}\left(A^{1}+\mathrm{i} A^{2}\right) \otimes \mathbb{1}_{3} & \mathrm{i}\left(-A^{0}-A^{3}\right) \otimes \mathbb{1}_{3} & \mathrm{i} \gamma^{5} \bar{\Phi}_{1} \otimes M_{\nu} & -\mathrm{i} \gamma^{5} \Phi_{2} \otimes M_{e} \\
\hline-\mathrm{i} \gamma^{5} \Phi_{2} \otimes M_{\nu}^{*} & \mathrm{i} \gamma^{5} \Phi_{1} \otimes M_{\nu}^{*} & 0_{3} & 0 \\
\hline-\mathrm{i} \gamma^{5} \bar{\Phi}_{1} \otimes M_{e}^{*} & -\mathrm{i} \gamma^{5} \bar{\Phi}_{2} \otimes M_{e}^{*} & 0 & -2 \mathrm{i} A^{0} \otimes \mathbb{1}_{3}
\end{array}\right),
\end{aligned}
$$

where $\mathbf{A} \in \Lambda^{1} \otimes \operatorname{su}(3), \quad \tilde{\mathbf{A}}:=\left(\begin{array}{lr}\mathrm{i} A^{3} ; & \mathrm{i}\left(A^{1}-\mathrm{i} A^{2}\right) \\ \mathrm{i}\left(A^{1}+\mathrm{i} A^{2}\right) ; & -\mathrm{i} A^{3}\end{array}\right) \in \Lambda^{1} \otimes \mathrm{su}(2), A^{0} \in \Lambda^{1}$, $\Phi_{1}, \Phi_{2} \in \Lambda^{0} \otimes \mathbb{C}$. In formula (7) for the curvature note that $\hat{\sigma}\left(\omega^{1}\right)=0$ $\bmod \hat{\pi}\left(\mathcal{J}^{2} \mathfrak{a}\right)$. Inserting (20) into (7) we obtain for the bosonic action given in (8)

$$
\begin{aligned}
& S_{B}=\frac{1}{48 g_{0}^{2}} \int_{X} d x \operatorname{tr}\left(\mathfrak{e}(\theta)^{2}\right)=\int_{X} d x\left(\mathscr{L}_{2}+\mathscr{L}_{1}+\mathscr{L}_{0}\right) \\
& \mathscr{L}_{2}=\frac{1}{4 g_{0}^{2}} \operatorname{tr}\left(\left(\mathbf{d} \mathbf{A}+\frac{1}{2}\{\mathbf{A}, \mathbf{A}\}\right)^{2}\right)+\frac{1}{4 g_{0}^{2}} \operatorname{tr}\left(\left(\mathbf{d} \tilde{\mathbf{A}}+\frac{1}{2}\{\tilde{\mathbf{A}}, \tilde{\mathbf{A}}\}\right)^{2}\right)+\frac{5}{6 g_{0}^{2}} \operatorname{tr}\left(\left(\mathbf{d} A^{0}\right)^{2}\right) \\
& \begin{aligned}
\mathscr{L}_{1}=\frac{1}{24 g_{0}^{2}} \operatorname{tr}\left(\left|\mathbf{d} \Phi_{1}+\mathrm{i}\left(A^{0}+A^{3}\right) \Phi_{1}+\mathrm{i}\left(A^{1}-\mathrm{i} A^{2}\right)\left(\Phi_{2}+1\right)\right|^{2}+\right. \\
\left.+\left|\mathbf{d} \Phi_{2}+\mathrm{i}\left(A^{0}-A^{3}\right)\left(\Phi_{2}+1\right)+\mathrm{i}\left(A^{1}+\mathrm{i} A^{2}\right) \Phi_{1}\right|^{2}\right) \times \\
\quad \times \operatorname{tr}\left(3 M_{u} M_{u}^{*}+3 M_{d} M_{d}^{*}+M_{\nu} M_{\nu}^{*}+M_{e} M_{e}^{*}\right) \\
\mathscr{L}_{0}=\frac{1}{192 g_{0}^{2}}\left(\left|\Phi_{1}\right|^{2}+\left|\Phi_{2}+1\right|^{2}-1\right)^{2} \operatorname{tr}(1) \times \\
\times \operatorname{tr}\left(6 \tilde{M}_{\{u d\}}^{2}+12 \tilde{M}_{u u}^{2}+12 \tilde{M}_{d d}^{2}+2 \tilde{M}_{\{\nu e\}}^{2}+4 \tilde{M}_{\nu \nu}^{2}+4 \tilde{M}_{e e}^{2}\right)
\end{aligned}
\end{aligned}
$$

We perform the reparameterizations

$$
\begin{aligned}
& \mathbf{A}=\sum_{a=1}^{8} \frac{\mathrm{i} g_{0}}{2} G_{\mu}^{a} \gamma^{\mu} \otimes \lambda^{a}, \tilde{\mathbf{A}}=\sum_{a=1}^{3} \frac{\mathrm{i} g_{0}}{2} W_{\mu}^{a} \gamma^{\mu} \otimes \sigma^{a}, A^{0}=\frac{\mathrm{i} g_{0}}{2} \sqrt{\frac{3}{5}} W_{\mu}^{0} \gamma^{\mu}, \\
& \Phi_{i}=g_{0} \phi_{i} / \sqrt{\operatorname{tr}\left(M_{u} M_{u}^{*}+M_{d} M_{d}^{*}+\frac{1}{3} M_{\nu} M_{\nu}^{*}+\frac{1}{3} M_{e} M_{e}^{*}\right)}, i=1,2,
\end{aligned}
$$

where $\left\{\sigma^{a}\right\}$ are the Pauli matrices and $\left\{\lambda^{a}\right\}$ the Gell-Mann matrices. Using

$$
\operatorname{tr}\left(\left(\gamma^{\kappa} \wedge \gamma^{\lambda}\right)\left(\gamma^{\mu} \wedge \gamma^{\nu}\right)\right)=4\left(\delta^{\lambda \mu} \delta^{\kappa \nu}-\delta^{\kappa \mu} \delta^{\lambda \nu}\right), \operatorname{tr}\left(\gamma^{\mu} \gamma^{\nu}\right)=4 \delta^{\mu \nu}, \operatorname{tr}(1)=4
$$

and performing a Wick rotation to Minkowski space we obtain for (21) precisely the bosonic action of the standard model, see [12]. Here, the Weinberg angle $\theta_{W}$ and the masses $m_{W}, m_{Z}$ and $m_{H}$ of the $W, Z$ and Higgs bosons are given by

$$
\begin{aligned}
m_{W} & =\frac{1}{2} \sqrt{\operatorname{tr}\left(M_{u} M_{u}^{*}+M_{d} M_{d}^{*}+\frac{1}{3} M_{\nu} M_{\nu}^{*}+\frac{1}{3} M_{e} M_{e}^{*}\right)}=\frac{1}{2} m_{t}, \\
m_{Z} & =m_{W} / \cos \theta_{W}, \sin ^{2} \theta_{W}=\frac{3}{8} \\
m_{H} & =\sqrt{\frac{\operatorname{tr}\left(\tilde{M}_{\{u d\}}^{2}+2 \tilde{M}_{u u}^{2}+2 \tilde{M}_{d d}^{2}+\frac{1}{3} \tilde{M}_{\{\nu e\}}^{2}+\frac{2}{3} M_{\nu \nu}^{2}+\frac{2}{3} M_{e e}\right)}{\operatorname{tr}\left(M_{u} M_{u}^{*}+M_{d} M_{d}^{*}+\frac{1}{3} M_{\nu} M_{\nu}^{*}+\frac{1}{3} M_{e} M_{e}^{*}\right)}}=\frac{3}{2} m_{t},
\end{aligned}
$$


where $m_{t}$ is the mass of the top quark. Here we have neglected the other fermion masses against $m_{t}$. The analogous relations in non-commutative geometry read for the simplest scalar product [6]

$$
m_{W}=\frac{1}{2} m_{t}, \quad m_{H}=\sqrt{\frac{69}{28}} m_{t}, \quad \sin ^{2} \theta_{W}=\frac{12}{29} .
$$

Inserting (20) and (22) into the fermionic action in (8) we arrive after a Wick rotation to Minkowski space and imposing the chirality condition $\Gamma h=h$ at the usual fermionic action of the standard model [12].

\section{REFERENCES}

[1] A. Connes, Non commutative geometry, Academic Press, New York 1994.

[2] A. Connes and J. Lott, The Metric Aspect of Noncommutative Geometry, Proceedings of 1991 Cargèse Summer Conference, ed. by J. Fröhlich et al, Plenum, New York 1992.

[3] A. Connes, Noncommutative geometry and reality, J. Math. Phys. 36 (1995) 6194-6231.

[4] B. Iochum, D. Kastler and T. Schücker, Fuzzy Mass Relations in the Standard Model, preprint hep-th/9507150.

[5] C. P. Martín, J. M. Gracia-Bondía and J. C. Varilly, The standard model as a noncommutative geometry: the low energy regime, preprint hep-th/9605001.

[6] D. Kastler and T. Schücker, The Standard Model à la Connes-Lott, preprint hepth/9412185.

[7] D. Kastler and T. Schücker, A Detailed Account of Alain Connes' Version of the Standard Model in Non-Commutative Differential Geometry IV, Rev. Math. Phys. 8 (1996) 205-228.

[8] R. Coquereaux, G. Esposito-Farèse and G. Vaillant, Higgs Fields as Yang-Mills Fields and Discrete Symmetries, Nucl. Phys. B 353 (1991) 689-706.

[9] R. Coquereaux, G. Esposito-Farèse and F. Scheck, Noncommutative Geometry and Graded Algebras in Electroweak Interactions, Intern. J. Mod. Phys. A 7 (1992) 6555-6593.

[10] R. Wulkenhaar, The Mathematical Footing of Non-associative Geometry, preprint hepth/9607094.

[11] F. Lizzi, G. Mangano, G. Miele and G. Sparano, Constraints on Unified Gauge Theories from Noncommutative Geometry, preprint hep-th/9603095.

[12] M. Guidry, Gauge Field Theories, Wiley, New York (1991). 